\title{
Multiobjective Algorithms Hybridization to Optimize Broadcasting Parameters in Mobile Ad-Hoc Networks
}

\author{
Sandra García, Cristóbal Luque, Alejandro Cervantes, and Inés M. Galván \\ Computer Science Departament, Carlos III University of Madrid \\ Avda. Universidad 30, 28911 Leganes, Spain \\ \{sgrodrig, cluque, acervant, igalvan\}@inf.uc3m.es
}

\begin{abstract}
The aim os this paper is to study the hybridization of two multi-objective algorithms in the context of a real problem, the MANETs problem. The algorithms studied are Particle Swarm Optimization (MOPSO) and a new multiobjective algorithm based in the combination of NSGA-II with Evolution Strategies (ESN). This work analyzes the improvement produced by hybridization over the Pareto's fronts compared with the non-hybridized algorithms. The purpose of this work is to validate how hybridization of two evolutionary algorithms of different families may help to solve certain problems together in the context of MANETs problem. The hybridization used for this work consists on a sequential execution of the two algorithms and using the final population of the first algorithm as initial population of the second one.
\end{abstract}

\section{Introduction}

Nowadays, many real-world optimization problems require compromise between several objectives. Mathematically, this is modelled as trying to simultaneously optimize several fitness functions depending on the same set of parameters.

Whenever there is a chance of combination of these objectives in a single objective function, these problems can be solved using conventional single-objective optimization methods. However, quite often there is no easy, or proper, way to combine the problem's objectives; this happens whenever objectives are conflicting, that is, improving some of the objectives means decreasing some of the rest. In these cases we need to solve a Multiobjective Optimization problem. The solution to this kind of problems is known as the pareto-optimal solution [2].

Multiobjective Evolutionary Algorithms (MOEAs) have been proved to be specially suited for this kind of optimization task. Some of the better known are SPEA 3] , PAES [4] or NSGA-II [5]. Hybrid metaheuristics are currently a subject of great interest for the community. Some combinations of techniques have been quite successful, such as combination of Local Search (LS) heuristics (Hill-descent, Simulated Annealing, Tabu Search) or even combinations of a LS technique with Evolutionary-based techniques. There are also some tools that may help performing this kind of experiments: for instance, Paradiseo $[6]$ or Team 7 . 
In this paper we consider the problem of broadcasting on a particular sub-class of MANETs called Metropolitan MANETs (Mobile Ad-hoc Networks). This problem has been already studied using Multiobjective Evolutionary Algorithms [8].

The purpose of this work is to study how hybridization of two evolutionary algorithms of different families may help to improve the results obtained by individual algorithm for the MANETs problem. The evolutionary multi-objective algorithms combined in this work are MOPSO (Multi-Objective Particle Swarm Optimization) and ESN (Evolution Stratety with NSGAII) algorithms 9]. The first one is an adapted version of Particle Swarm Optimization to multi-objective optimization problems. And the second one is a multi-objective version for the Evolutionary Strategy algorithm.

\section{MANETs Problem Definition}

According to IETF (Internet Engineering Task Force), MANETs are autoconfiguration structures where stations (also called terminals) are temporally connected without a pre-existing infrastructure or a centralized administration 10 . MANETs are wireless networks where one station can communicate directly with the other stations.

In the last years, MANETs have increased its interest, as path to the $3 \mathrm{G}$ networks: a) they allow to extend the coverage, b) they don't need new infrastructure. In this way, a terminal user can use another users' terminals as part of a multistep path toward the kernel of the network.

In this paper we consider the problem of broadcasting on a particular sub-class of MANETs called Metropolitan MANETs, which span from shopping malls to metropolitan areas. Instead of providing a generic set of parameters that performs well on average situations, the goal is to optimize the broadcast messages. Optimizing a broadcasting strategy is a multiobjective problem where multiple functions have to be satisfied at the same time.

For this work, the broadcasting strategy considered for optimization is DFCN 11. The DFCN strategy defines five parameters (minGain, lowerBoundRAD, upperBoundRAD, proD and safeDensity) to determine the following three objective values:

- Minimizing the makespan, or time required to perform the broadcast.

- Maximizing the network coverage, or number of stations reached.

- Minimizing the bandwidth used for broadcast messages and retries.

These three objectives define the objective space, and are considered three independent fitness functions. The purpose of optimization is to tune the input parameters of DFCN in order to find the three-dimensional Pareto-optimal front defined in objective space. The front is made by all the feasible non-dominated values for the three objective functions. 


\section{Multi-Objective Algorithms}

The hybridization studied in this work consists on just sequentially executing the two algorithms and using the output population of the first algorithm to initialize the population of the second one. Thus, one algorithm is executed during a number of generations and, afterwards, the other algorithm is executed using as initial population the result obtained by the first one. This mix of populations requires certain considerations because individuals of the algorithms use different information. For MOPSO, individuals represent a particle with a position in the swarm and a velocity. However, ESN works with individuals that have assigned a variance value, which is updated during the evolution and it is used to generate new individuals. Thus, depending on the order in which the algorithms are executed, the following considerations are taken into account:

- MOPSO+ESN: When the population obtained by MOPSO is used to initialize ESN, the position of particles are the individuals for ESN and the variance value for all individuals in the population is fixed to 0.1 .

- ESN+MOPSO: When the first algorithm used is ESN and the population obtained after a number of generations is used to initialize the swarm, the position of particles is initialized using the solutions of ESN and the velocity is determined randomly from the interval $[-1.0,1.0]$

\subsection{Multi-Objective Particle Swarm Optimization: MOPSO}

MOPSO [1 is an adapted version of Particle Swarm Optimization (PSO) to multi-objective optimization problems. MOPSO combines PSO [12 with the archiving strategy of PAES 4. In this work, we use the version available in the EMOO repository [13].

The algorithm uses an external repository that stores non-dominated solutions in the swarm. Each iteration, for each particle, a leader is selected. The mechanism of leader selection is as follows: the fitness space is divided in hypercubic sectors, and one of the positions in the repository is randomly selected using a roulette algorithm that favors the sectors that are less populated. The velocity and position of particles are updated using the standard equations of PSO but using the leader particle selected from the repository. The fitness of the new position is calculated. This new position is compared with solutions already in the repository, and is inserted if it is a non-dominated solution. The maximum number of particles in the repository is fixed, so when this limit is reached, before the insertion, a particle from the most-populated sector of the repository is removed. If the new solution dominates some of the solutions in the repository, those solutions are removed.

\subsection{Evolution Stratety with NSGAII: ESN}

The ESN algorithm is based on the hybridization of Evolution Strategies and NSGAII. The algorithm uses the standard Evolution Strategies' steps [14], replacing 
the selection process by the NSGAII [5] selection process. In our implementation of Evolution Strategies, each of the $\mu$ individuals in the population produces one offspring only by mutation. From the resulting population of $2 \cdot \mu$ individuals, the best $\mu$ are selected for the next generation.

In the reproduction phase, for each individual the variance value is updated. Each parent generates one child using the new variance and a Gaussian distribution. After reproduction, the population is sorted using the NSGAII mechanism. This process creates the front $F_{1}$ with all the non-dominated solutions in the population. Then, those individuals are discarded and new fronts are created using the rest of the population. The worst $\mu$ individuals are deleted using the following criterion: an individual is better than another when it belongs to a front with a lower index; if two individuals are in the same front, the one with the greater crowding distance is better.

\subsection{Results Evaluation: Metrics for Multiobjective Problems}

Solutions resulting from different executions of the multiobjective algorithms must be compared using quantitative metrics that measure the success of the algorithms toward the MO problem objectives: distance to the "true" Paretooptimal front, distribution of solutions over the obtained front, and spread.

It is generally admitted that there is no single metric that can be used to evaluate those objectives simultaneously; this is specially true when the best Pareto-optimal front is not known. A detailed description of available metrics can be found in [15].

We have chosen the following metrics to compare solutions (fronts) obtained by the algorithms:

- Set Coverage (SC). Coverage of a set of points A over a set of points B $(S C(A, B))$ is defined as the fraction of the points in set $\mathrm{B}$ that are weakly dominated by a point in set $\mathrm{A}$. This measure has to be calculated in both directions (A vs. $\mathrm{B}$ and $\mathrm{B}$ vs. A) because $S C(A, B) \neq 1-S C(B, A)$.

- Hypervolume (HV). This metric, also called size of the dominated space, is the volume enclosed by the union of the of the points in the set. The volume dominated by any point is calculated as the volume of the hypercube defined by each point. The value of this metric is usually calculated after normalization of the points in the solutions.

Set Coverage is a measure of how a set dominates the other in terms of number of points. However, it does not take into account the actual distance between the points in both sets; that is, "how much" a point in a set dominates the rest. It requires two sets of points (two fronts) for comparison, so it cannot be used to assign a performance measure to a single front except if the "true" Paretooptimal front is known and used as reference.

Hypervolume assigns a quantitative value to a given set (where greater values mean better performance) but it cannot be used to derive dominance of a set over the other and its actual value depends on the normalization used. 


\section{Experiments}

In this section we present the utility of algorithm hybridization proposed in this article in order to solve the aforementioned problem, tuning of the parameteres of the DFCN broadcasting protocol for MANETs.

All the experiments are performed using Madhoc [16], a Metropolitan MANET simulator. It aims at providing a tool for simulating different level services based on distinct technologies on MANETs for different environments. The simulator provides several different models of network structure, of which we have used the Mall environment, that simulates a commercial shopping center.

\subsection{Experimental Settings}

Experiments have been performed using different combinations of the order of execution of the algorithms and the number of evaluations performed by each of the algorithms.

Moreover, we have also executed experiments using only MOPSO and ESN, and we have calculated their metrics in order to compare their results with the other obtained by the hybridizations. The obtained fronts have been evaluated using the metrics explained in section 3.3 and their results are shown in tables 123 and 4

All of these experiments used a total of 25000 evaluations. The population size for both algorithms was 100 individuals/particles. The initial variance for ESN was 0.1 , and the value of the constant $\Delta$ was set to 0.7 .

MOPSO used a swarm size of 100 particles, and fixed PSO parameters: $w=$ $0.4, r_{1}=r_{2}=2.0, X=0.4$. We used 30 divisions of the adaptive grid as suggested in [1]. The number of non-dominated points in the solution was limited to 100 .

In tables presented in this section, ESN- $X+$ MOPSO- $Y$ means an experiment of $X$ evaluations of the ESN algorithm, followed by $Y$ evaluations of the MOPSO algorithm (and thus $X+Y=25000$ ). A generation of 100 individuals takes 100 evaluations (one for each indivivual). So, $X$ evaluations represents $X / 100$ generations. In the same way for MOPSO, $Y$ evaluations represents $Y / 100$ iterations.

For each configuration, five experiments were performed. Tables in this section shown the mean value of the metrics for those experiments.

\subsection{Experimental Results}

Table 1 presents the Hypervolume for the different combinations of algorithms. As we can see, all the solutions have very similar values for this metric. The best result is attained by ESN-25000, but it is not much better than the other results. Thus, we can conclude that this metric is not enough to compare the quality of the hybrid algorithms, so we need to study the Set Coverage.

Set Coverage is studied in tables 2, 3] and 4, Table 22 shows the dominance that each hybridization obtain over the solutions that the MOPSO and ESN nonhybridized algorithms produces for this problem. Table 3 shows the dominance 
Table 1. Hypervolume

\begin{tabular}{lcc}
\hline Algorithm & Hypervolume & Variance \\
\hline ESN-8000 + MOPSO-17000 & 0.7123 & 0.0000 \\
ESN-12500 + MOPSO-12500 & 0.7119 & 0.0000 \\
ESN-17000 + MOPSO-8000 & 0.7108 & 0.0000 \\
MOPSO-8000 + ESN-17000 & 0.7143 & 0.0000 \\
MOPSO-12500 + ESN-12500 & 0.7116 & 0.0000 \\
MOPSO-17000 + ESN-8000 & 0.7080 & 0.0000 \\
MOPSO-25000 & 0.7080 & 0.0000 \\
ESN-25000 & 0.7195 & 0.0002 \\
\hline
\end{tabular}

Table 2. Set Coverage

\begin{tabular}{lcccc}
\hline Set Coverage & MOPSO-25000 & Variance & ESN-25000 & Variance \\
\hline ESN-8000 + MOPSO-17000 & 0.4267 & 0.0082 & 0.3461 & 0.0044 \\
ESN-12500 + MOPSO-12500 & 0.4444 & 0.0132 & 0.3576 & 0.0062 \\
ESN-17000 + MOPSO-8000 & 0.4420 & 0.0104 & 0.3774 & 0.0057 \\
MOPSO-8000 + ESN-17000 & 0.4351 & 0.0087 & 0.3614 & 0.0083 \\
MOPSO-12500 + ESN-12500 & 0.4168 & 0.0081 & 0.3362 & 0.0030 \\
MOPSO-17000 + ESN-8000 & 0.4034 & 0.0094 & 0.3052 & 0.0063 \\
MOPSO-25000 & - & - & 0.3548 & 0.0092 \\
ESN-25000 & 0.4581 & 0.0153 & - & - \\
\hline
\end{tabular}

Table 3. Set Coverage

\begin{tabular}{lcccccc}
\hline Set Coverage & $\begin{array}{c}\text { ESN-8000 }+ \\
\text { MOPSO-17000 }\end{array}$ & Variance & ESN-12500 + & \multicolumn{3}{c}{ ESN-17000 + } \\
& MOPS-12500 & Variance & MOPSO-8000 Variance \\
\hline MOPSO-25000 & 0.3552 & 0.0124 & 0.3436 & 0.0093 & 0.3368 & 0.0101 \\
ESN-25000 & 0.4464 & 0.0115 & 0.4332 & 0.0102 & 0.4348 & 0.0152 \\
\hline
\end{tabular}

Table 4. Set Coverage

\begin{tabular}{lcccccc}
\hline Set Coverage & \multicolumn{1}{c}{ MOPSO-8000 } & \multicolumn{3}{c}{ MOPSO-12500 } & \multicolumn{3}{c}{ MOPSO-17000 } \\
& + ESN-17000 & Variance & + ESN-12500 & Variance & + ESN-8000 & Variance \\
\hline MOPSO-25000 & 0.3370 & 0.0065 & 0.3636 & 0.0095 & 0.3708 & 0.0124 \\
ESN-25000 & 0.4726 & 0.0196 & 0.4608 & 0.0130 & 0.5164 & 0.0145 \\
\hline
\end{tabular}

of the non-hybridized algorithms over the ESN+MOPSO hybridized algorithms, and table 4 over the MOPSO+ESN algorithms.

Next, we analyze the Set Coverage obtained by the execution using first Evolution Strategy with NSGA-II as a first part and Particle Swarm Optimization as a second part of the hybridization. If we compare the percent of dominant points of these first three approaches versus MOPSO, we can see how these hybridizations improve the Set Coverage obtained by the solutions of "MOPSO25000". But this does not happen with "ESN-25000" because their Pareto fronts are better (they have a greater Set Coverage) than the solutions obtained by the hybrid algorithm, as can be seen in tables 3 and 4 . In conclusion, we cannot say that the hybridization in which ESN starts the execution improves the solutions obtained by the executions of the non-hybridized algorithms.

Continuing with the Set Coverage analysis, the experiments which use MOPSO as the first algorithm and ESN as the second one, we can see that the more ESN evaluations we perform, the better Set Coverage it obtains over the non-hybridized ESN. This also happens when we compare the hybridized experiments with nonhybridized MOPSO: it means that using more ESN evaluations produces better 
Set Coverage over non-hybridized MOPSO. We think this is due to fact that the ESN algorithm has better performance in this kind of problem and helps MOPSO to find better fronts.

For the experiments with hybridized algorithms, we usually find better fronts in terms of Set Coverage when ESN is used first; and when MOPSO is used first, the results are better as long as MOPSO has less evaluations.

\section{Conclusions}

A mobile ad-hoc network (MANETs) is defined as a self-configuring network of mobile routers (and associated hosts). Due to the fact that network topology varies dynamically, the operations of broadcasting are of utter importance for the existence and the performance of the network and can benefit from parameter optimization on the broadcasting algorithm used in the network.

We have defined the optimization problem for the broadcasting strategy in MANETs as a multiobjective problem where a single solution is defined as a set of values for those parameters, and the objectives that must be simultaneously achieved are: Maximum Coverage, Minimum Bandwith usage and Minimum makespan. Experiments are conducted by simulation of the broadcasting protocol in a predefined network environment.

This paper applies the hybridization of two different MOEAs (MOPSO and ESN) to find the Pareto-optimal front, that is, the set of non-dominated solutions for this problem. In order to study the benefits of the hybridization, experiments using the non-hybridized algorithms, MOPSO and ESN, were also carried out. All experiments were compared using two standard metrics for evaluation of the solutions to multiobjective problems: Set Coverage and Hypervolume.

Results show that both algorithms and their different combinations are able to find non-dominated fronts that approximate the "true" Pareto-optimal front with adequate accuracy and distribution of the solutions.

When comparing the hybridizations, in terms of Hypervolume we cannot decide what performance is the best one, because its results are very similar. In terms of Set Coverage, we have proved that hybridization is a good tool to improve the results of MOPSO, but ESN without any hybridization obtains better fronts than other combinations of MOPSO and ESN.

Acknowledgments. This article has been financed by the Spanish founded research MEC projects OPLINK::UC3M, Ref:TIN2005-08818- C04-02 and MSTAR::UC3M, Ref:TIN2008-06491-C04-03.

\section{References}

1. Coello, C.A., Toscano, G., Salazar, M.: Handling multiple objectives with particle swarm optimization. IEEE Transactions on Evolutionary Computation 8(3), 256279 (2004)

2. Goldberg, D.: Genetic algorithms in Sarch, Optimization, and Machine Learnig. Addison Wesley Publishing Company, Reading (1989) 
3. Zitzler, E., Laumanns, M., Thiele, L.: SPEA2: Improving the Strength Pareto Evolutionary Algorithm for Multiobjective Optimization, EUROGEN, pp. 95-100 (2001)

4. Knowles, J.D., Corne, D.W.: Approximating the nondominated front using the pareto archived evolution strategy. Evol. Comput. 8(2), 149-172 (2000)

5. Deb, K., Pratap, A., Agarwal, S., Meyarivan, T.: A Fast and Elitist Multiobjective Genetic Algorithm: NSGA-II. IEEE Transactions on Evolutionary Computation 6(2), 182-197 (2002)

6. Talbi, E., Cahon, S., Melab, N.: ParadisEO: A Framework for the Reusable Design of Parallel and Distributed Metaheuristics. Journal of Heuristics, 357-380 (2004)

7. León, C., Miranda, G., Segura, C.: A Parallel Plugin-Based Framework for Multiobjective Optimization. In: International Symposium on Distributed Computing and Artificial Intelligence, vol. 50/2009, pp. 142-151 (2008)

8. Alba, E., Dorronsoro, B., Luna, F., Nebro, A.J., Bouvry, P., Hogie, L.: A Cellular Multi-Objective Genetic Algorithm for Optimal Broadcasting Strategy in Metropolitan MANETs. Computer Communications 30(4), 685-697 (2007)

9. Pérez Pérez, R., Luque, C., Cervantes, A., Isasi, P.: Multiobjective Algorithms to Optimize Broadcasting Parameters in Mobile Ad-hoc Networks. In: IEEE Congress on Evolutionary Computation, pp. 3142-3149 (2007)

10. IETF Secretariat: IEFT MANET Charter (2007), http://www.ietf.org/html.charters/manet-charter.html

11. Hogie, L., Guinand, F., Bouvry, P.: A Heuristic for Efficient Broadcasting in the Metropolitan Ad Hoc Network. In: 8th Int. Conf. on Knowledge-Based Intelligent Information and Engineering Systems, pp. 727-733 (2004)

12. Kennedy, J., Eberhart, R.C., Shi, Y.: Swarm intelligence. Morgan Kaufmann Publishers, San Francisco (2001)

13. Coello, C.A., et al.: EMOO Repository, http://www.lania.mx/ccoello/EMOO

14. Bäck, T., Schwefel, H.P.: Evolutionary Algorithms: Some Very Old Strategies for Optimization and Adaptation. New Computing Techniques in Physics Research II. In: Proc. Second Int'l Workshop Software Engineering, Artificial Intelligence, and Expert Systems for High Energy and Nuclear Physics, pp. 247-254 (1992)

15. Zitzler, E., Thiele, L.: An Evolutionary Algorithm for Multiobjective Optimization: The Strength Pareto Approach, Technical Report 43, Gloriastrasse 35, CH- 8092 Zurich, Switzerland, http://citeseer.ist.psu.edu/article/zitzler99evolutionary.html (1998)

16. Hogie, L., Guinand, F., Bouvry, P.: The madhoc metropolitan adhoc network simulator. Technical report, Université du Luxembourg and Université du Havre, France (March 2006), http://wwwlih.univ-lehavre.fr/hogie/madhoc/ 